\title{
Rendimentos de carcaça, cortes comerciais e não-componentes da carcaça de cordeiros Santa Inês alimentados com feno de erva-sal e concentrado
}

\author{
Carcass yields, commercial cuts and non-carcass components of Santa Ines lambs fed \\ with saltbush hay and concentrate
}

MORENO, Greicy Mitzi Bezerra ${ }^{1 *}$; BORBA, Hirasilva²; ARAÚJO, Gherman Garcia Leal de ${ }^{3}$; VOLTOLINI, Tadeu Vinhas ${ }^{3}$; SOUZA, Rafael Araújo ${ }^{4}$; SILVA SOBRINHO, Américo Garcia da ${ }^{5}$; BUZANSKAS, Marcos Eli ${ }^{6}$; LIMA JÚNIOR, Dorgival Morais $\mathrm{de}^{1}$; ALVARENGA, Tharcilla Isabella Rodrigues Costa $^{7}$

\footnotetext{
${ }^{1}$ Universidade Federal de Alagoas, Campus Arapiraca, Arapiraca, Alagoas, Brasil.

${ }^{2}$ Universidade Estadual Paulista, Departamento de Tecnologia, Jaboticabal, São Paulo, Brasil.

${ }^{3}$ Embrapa Semiárido, Petrolina, Pernambuco, Brasil.

${ }^{4}$ Universidade Federal da Bahia, Escola de Medicina Veterinária e Zootecnia, Programa de PósGraduação em Zootecnia, Salvador, Bahia, Brasil.

${ }^{5}$ Universidade Estadual Paulista, Departamento de Zootecnia, Jaboticabal, São Paulo, Brasil.

${ }^{6}$ Universidade Estadual Paulista, Departamento de Ciências Exatas, Jaboticabal, São Paulo, Brasil.

${ }^{7}$ Universidade Federal de Lavras, Lavras, Minas Gerais, Brasil.

*Endereço para correspondência: greicymitzimoreno@yahoo.com.br
}

\section{RESUMO}

Objetivou-se com este trabalho avaliar os rendimentos de carcaça, cortes comerciais e não-componentes da carcaça de cordeiros Santa Inês alimentados com $30,40,50$ e $60 \%$ de feno de erva-sal associado a concentrado. Foram utilizados 32 ovinos machos castrados, com 8 meses de idade e peso inicial de $22 \pm 1,97 \mathrm{~kg}$, confinados individualmente e abatidos após 60 dias de confinamento. Os pesos de carcaça quente e fria decresceram linearmente com o aumento de feno de erva-sal na dieta, no entanto, não houve efeito para o rendimento verdadeiro de carcaça $(59,42 \%)$ e dos cortes: paleta $(19,26 \%)$, pescoço $(8,52 \%)$, costelas $(26,57 \%)$ e perna $(33,88 \%)$. Houve efeito linear decrescente para área de olho de lombo e espessura de gordura à medida que o nível de feno de erva-sal aumentou na dieta. Os pesos e os rendimentos de pele, fígado, coração, rins com gordura perirrenal e gorduras omental e mesentérica decresceram linearmente com a inclusão de feno de erva-sal na dieta, enquanto o conteúdo (\%) do trato gastrintestinal aumentou. $\mathrm{O}$ aumento da proporção de feno de erva-sal na dieta de cordeiros proporciona adequados rendimentos verdadeiros de carcaça e dos cortes comerciais, no entanto, reduz a área de olho de lombo e a espessura de gordura subcutânea, medidas importantes na avaliação qualitativa da carcaça ovina.

Palavras-chave: área de olho de lombo, confinamento, plantas halófitas, ovinos, semiárido

\section{SUMMARY}

This work aimed to evaluate the carcass yields, commercial cuts and non-carcass components of Santa Inês lambs fed with $30,40,50$ or $60 \%$ of oldman saltbush hay with concentrated. Were used 32 castrated lambs at 8 months old and initial body weight of $22 \pm 1.97 \mathrm{~kg}$, housed individually and slaughtered after 60 days of feedlot. The weights of hot and cold carcass decreased linearly with the inclusion of saltbush hay in the diet, however, there was no effect for true carcass yield and commercial cuts: shoulder $(19.26 \%)$, neck $(8.52 \%)$ ribs $(26.57 \%)$ and leg $(33.88 \%)$. There was a linear decrease for ribeye area and fat thickness when the level of hay in the diet increased. The weights and yields of skin, liver, heart, kidneys with perirenal fat, omental and mesenteric fats decreased linearly 
with the inclusion of hay in the diet, while the content (\%) of gastrointestinal tract increased. The increased proportion of saltbush hay in the diet of lambs provided adequate true carcass yield and commercial cuts, however, reduces the ribeye area and fat thickness, important measurements in the qualitative evaluation of the lamb's carcass.

Keywords: feedlot, halophyte plants, ribeye area, semiarid, sheep

\section{INTRODUÇÃO}

As mudanças climáticas que vêm ocorrendo nas últimas décadas estão acarretando em aumento das temperaturas e redução das precipitações, o que tem contribuído na expansão da salinização dos solos e da água em diversos países do mundo, especialmente em regiões áridas e semiáridas. Esta realidade tem ressaltado $\mathrm{o}$ interesse de produtores $\mathrm{e}$ pesquisadores por plantas resistentes ou tolerantes à salinidade (halófitas), bem como à seca (xerófitas), de forma que se possa produzir alimentos para os animais em condições adversas de solo e clima (Van NIEKERK et al., 2009; EL SHAER, 2010; MENESES et al., 2012). Dentre as halófitas cultiváveis para fins forrageiros, as espécies do gênero Atriplex L. merecem destaque especial, em virtude de sua rusticidade e capacidade de crescimento em áreas altamente salinas e com baixos índices pluviométricos (BEN SALEM et al., 2010; EL SHAER, 2010; PEARCE et al., 2010), podendo contribuir no fornecimento de alimentos no confinamento e garantir a produção de carne de forma sustentável.

Algumas pesquisas desenvolvidas na Austrália revelaram que as carcaças de ovinos alimentados com erva-sal possuem menor teor de gordura subcutânea (PEARCE et al., 2008a; PEARCE et al., 2008b), o que constitui uma importante ferramenta de marketing para atender um mercado consumidor crescente que busca uma alimentação mais saudável com menor consumo de gordura. Este fato pode estar associado à baixa relação proteína:energia e a presença de alta quantidade do aminoácido betaína na erva-sal, que tem sido relacionado com redução da gordura subcutânea e intramuscular em cordeiros (FERNANDEZ et al., 2000). Também tem sido relatado que a erva-sal tem elevada concentração de cromo e que este componente estaria envolvido na redução da lipogênese e da deposição de gordura visceral e subcutânea (GARDNER et al., 1998). No entanto, as dietas de terminação devem garantir um teor mínimo de gordura nas carcaças, especialmente a subcutânea, para garantir a qualidade sensorial da carne e garantir mínimas perdas por resfriamento nas carcaças.

Sabendo que o nível nutricional de ovinos afeta a qualidade e os rendimentos das carcaças comercializadas, assim como a proporção dos cortes comerciais, são importantes estudos que avaliem o efeito do plano nutricional sobre estas características. Neste sentido, os objetivos deste estudo foram avaliar os rendimentos de carcaça, cortes comerciais e nãocomponentes da carcaça de cordeiros Santa Inês alimentados com 30, 40, 50 e $60 \%$ de feno de erva-sal e concentrado.

\section{MATERIAL E MÉTODOS}

O trabalho foi desenvolvido na Embrapa Semiárido, Petrolina (PE). Foram utilizados 32 cordeiros Santa Inês, castrados, com peso inicial médio de $22 \pm 1,97 \mathrm{~kg}$ e 8 meses de idade. Os animais foram identificados, everminados, vacinados 
contra Clostridioses e distribuídos aleatoriamente nos tratamentos, adotando-se 20 dias de adaptação às dietas experimentais e instalações. Os animais foram alojados em baias individuais, providas de comedouro, bebedouro e saleiro, e dispostas em área coberta.

Os tratamentos foram constituídos por dietas contendo $30 ; 40 ; 50$ e $60 \%$ de feno de erva-sal associado a alimento concentrado, perfazendo diferentes relações volumoso:concentrado. Os concentrados foram compostos por milho moído, farelo de soja e ureia, e as dietas calculadas para serem isoproteicas $(12,0 \%$ PB) e de acordo com as tabelas preconizadas pelo NRC (2007), para atender às exigências de ovinos de maturação tardia, objetivando ganhos de peso de $200 \mathrm{~g} /$ animal/dia.

$\mathrm{A}$ alimentação foi fornecida às $9 \mathrm{~h}$ e às $16 \mathrm{~h}$, com controle diário da quantidade fornecida, permitindo $20 \%$ de sobras. Adotou-se 60 dias de confinamento, em que os animais foram pesados no início (peso inicial) e no fim do experimento (peso final). Nas Tabelas $1 \mathrm{e}$ 2 podem ser visualizadas as composições bromatológica e percentual dos ingredientes e bromatológica das dietas, expressas na matéria seca.

Após 60 dias de confinamento, os cordeiros foram pesados antes e após o jejum de sólidos por 18 horas para determinação do peso corporal ao abate (PCA) e, posteriormente, insensibilizados com descarga elétrica de $330 \mathrm{~V}$ durante 12 segundos e abatidos pela secção das veias jugulares e artérias carótidas. Após a sangria e esfola, procedeu-se a evisceração, sendo os componentes pesados individualmente: trato gastrintestinal (TGI), sangue, pele, esôfago, fígado, coração, aparelho respiratório (pulmões + traqueia), cabeça, patas, baço, rins + gordura perirrenal, gordura omental (recobre os estômagos) e gordura mesentérica (recobre os intestinos).

Tabela 1. Composição bromatológica dos ingredientes das dietas experimentais (expressa na matéria seca)

\begin{tabular}{lccc}
\hline \multirow{2}{*}{ Nutriente (\%) } & \multicolumn{3}{c}{ Ingrediente } \\
\cline { 2 - 4 } & Feno de erva-sal & Milho moído & Farelo de soja \\
\hline Matéria seca (\%) & 89,65 & 87,51 & 89,35 \\
Matéria orgânica (\%) & 82,91 & 97,68 & 93,02 \\
Cinzas (\%) & 17,09 & 2,32 & 6,98 \\
Proténa bruta (\%) & 7,72 & 8,91 & 50,94 \\
Extrato etéreo (\%) & 1,26 & 5,38 & 0,88 \\
FDN cp (\%) & 59,84 & 19,30 & 25,00 \\
Fibra em detergente ácido (\%) & 41,84 & 6,69 & 13,02 \\
Celulose (\%) & 27,71 & 5,14 & 11,23 \\
Hemicelulose (\%) & 23,21 & 18,99 & 15,78 \\
Lignina (\%) & 14,13 & 1,54 & 1,79 \\
Carboidratos totais (\%) & 73,93 & 83,39 & 41,20 \\
Carboidratos não fibrosos (\%) & 14,09 & 64,09 & 16,19 \\
Energia bruta (Mcal/kg) & 3,66 & 4,45 & 4,64 \\
Sódio (\%) & 6,13 & 0,03 & 0,22 \\
Cloro (\%) & 7,51 & 0,04 & 0,08 \\
Fósforo (\%) & 0,48 & 0,67 & 0,80 \\
Magnésio (\%) & 0,34 & 0,12 & 0,53 \\
Potássio (\%) & 1,40 & 0,80 & 2,72 \\
\hline
\end{tabular}

Fibra em detergente neutro corrigido para cinzas e proteína. 
Rev. Bras. Saúde Prod. Anim., Salvador, v.15, n.1, p.192-205 jan./mar., 2014 http://www.rbspa.ufba.br ISSN 15199940

Tabela 2. Composição percentual dos ingredientes e bromatológica das dietas experimentais

\begin{tabular}{|c|c|c|c|c|}
\hline \multirow{2}{*}{ Composição } & \multicolumn{4}{|c|}{ Níveis de feno de erva-sal (Atriplex nummularia) } \\
\hline & $30 \%$ & $40 \%$ & $50 \%$ & $60 \%$ \\
\hline \multicolumn{5}{|l|}{ Percentual (\%) } \\
\hline Feno de erva-sal & 30 & 40 & 50 & 60 \\
\hline Milho moído & 59,15 & 49,50 & 39,90 & 30,30 \\
\hline Farelo de soja & 9,60 & 9,60 & 9,60 & 9,60 \\
\hline Ureia & 1,25 & 0,90 & 0,50 & 0,12 \\
\hline \multicolumn{5}{|l|}{ Bromatológica (na MS) } \\
\hline Matéria seca (\%) & 89,21 & 89,16 & 89,15 & 89,05 \\
\hline Matéria orgânica (\%) & 92,81 & 91,33 & 89,85 & 88,37 \\
\hline Cinzas (\%) & 7,19 & 8,67 & 10,15 & 11,63 \\
\hline Proteína bruta (\%) & 12,57 & 12,46 & 12,35 & 12,23 \\
\hline Extrato etéreo (\%) & 3,70 & 3,29 & 2,89 & 2,48 \\
\hline Fibra em detergente neutro (\%) & 37,47 & 41,49 & 45,53 & 49,57 \\
\hline FDN cp $(\%)^{\mathrm{a}}$ & 31,77 & 35,89 & 40,02 & 44,15 \\
\hline Fibra em detergente ácido (\%) & 17,83 & 21,35 & 24,87 & 28,39 \\
\hline Lignina (\%) & 5,34 & 6,60 & 7,86 & 9,12 \\
\hline Nutrientes digestíveis totais $(\%)^{\mathrm{b}}$ & 71,18 & 68,25 & 60,40 & 56,41 \\
\hline Energia bruta (Mcal/kg) & 4,23 & 4,15 & 4,07 & 3,99 \\
\hline
\end{tabular}

O TGI, constituído pelo rúmen, retículo, omaso, abomaso, intestino delgado e grosso, foi pesado cheio e vazio e, após esvaziamento completo e minuciosa lavagem, obteve-se por diferença o conteúdo do TGI. Posteriormente, obteve-se o peso do corpo vazio $(\mathrm{PCV}=$ PCA - conteúdo do trato gastrintestinal - conteúdo da bexiga - conteúdo da vesícula biliar), calculando-se as porcentagens dos não-componentes da carcaça em relação ao PCA.

Posteriormente, as carcaças foram pesadas (PCQ) para determinação do rendimento da carcaça quente $(\mathrm{RCQ}=$ PCQ/PCA x 100) e em seguida, foram transferidas para câmara frigorífica a $4^{\circ} \mathrm{C}$ por 24 horas, suspensas pelos tendões do gastrocnêmio, em ganchos apropriados para manter a distância de $17 \mathrm{~cm}$. As carcaças frias foram pesadas (PCF), calculando-se o rendimento de carcaça fria $(\mathrm{RCF}=\mathrm{PCF} / \mathrm{PCA} \times 100)$, a perda de peso por resfriamento $(\mathrm{PPR}=$ PCQ - PCF/PCQ x 100) e o rendimento verdadeiro (RV = PCQ/PCV x 100).

Posteriormente, as carcaças foram divididas longitudinalmente e a meia carcaça esquerda foi seccionada em cinco regiões anatômicas ou cortes comerciais sendo: pescoço, paleta, costelas, lombo e perna, segundo metodologia adaptada de ColomerRocher et al. (1988). Cada corte foi pesado separadamente e em seguida, calcularam-se suas porcentagens em relação à meia carcaça esquerda. Finalmente, procedeu-se às mensurações do músculo Longissimus lumborum, entre a $12^{\underline{a}}$ e $13^{\underline{a}}$ costelas, para determinação da área de olho de lombo (AOL), calculada pela fórmula $(\mathrm{A} / 2 \quad \mathrm{x} \quad \mathrm{B} / 2) \pi$, em que $\mathrm{A}$ é $\mathrm{o}$ comprimento máximo e $\mathrm{B}$ é a profundidade máxima do músculo, em cm (COSTA et al., 2012). Foram ainda 
determinadas as medidas $\mathrm{C}$ (espessura mínima de gordura de cobertura sobre o músculo Longissimus lumborum) e a medida GR (espessura máxima de gordura de cobertura sobre a superfície da $13^{\underline{a}}$ costela, a $11 \mathrm{~cm}$ da linha dorsolombar), obtidas com auxílio de um paquímetro digital e fita métrica.

O delineamento experimental utilizado foi o inteiramente casualizado com 4 tratamentos e 8 repetições. Os resultados foram avaliados por meio de análises de variância e regressão, em que os graus de liberdade foram desdobrados em efeito linear, quadrático ou cúbico, de acordo com os níveis de feno de erva-sal. A significância das regressões foi obtida pelo teste " $\mathrm{t}$ " a 1 ou $5 \%$ de probabilidade utilizando-se o programa estatístico SAS (STATISTICAL ANALYSIS SYSTEMS, 2002).

\section{RESULTADOS E DISCUSSÃO}

Os pesos e os rendimentos de carcaça quente e fria decresceram linearmente à medida que houve aumento do nível de feno de erva-sal na dieta (Tabela 3 ).

Tabela 3. Pesos corporal de abate (PCA), de carcaça quente (PCQ) e fria (PCF), perdas de peso por resfriamento (PPR), rendimentos de carcaça quente (RCQ), fria (RCF) e verdadeiro (RV) de cordeiros alimentados com níveis crescentes de feno de erva-sal e concentrado

\begin{tabular}{|c|c|c|c|c|c|c|c|c|c|}
\hline \multirow{2}{*}{ Variável } & \multicolumn{4}{|c|}{ Níveis de feno de erva-sal (\%) } & \multirow{2}{*}{$\begin{array}{l}\text { Equação de } \\
\text { regressão }\end{array}$} & \multicolumn{2}{|c|}{ Valor de $\mathrm{P}$} & \multirow{2}{*}{$\mathrm{R}^{2}$} & \multirow{2}{*}{$\begin{array}{l}\mathrm{CV} \\
(\%)\end{array}$} \\
\hline & 30 & 40 & 50 & 60 & & $\mathrm{~L}$ & Q & & \\
\hline $\begin{array}{l}\text { GPD } \\
\text { (g/dia) }\end{array}$ & 193,41 & 181,81 & 149,31 & 120,8 & 1 & 0,0044 & 0,6496 & 0,9683 & 31,85 \\
\hline PCA (kg) & 32,25 & 31,29 & 28,75 & 26,81 & 2 & 0,0050 & 0,7327 & 0,9740 & 13,23 \\
\hline PCQ (kg) & 16,75 & 15,56 & 13,94 & 13,00 & 3 & $<0,0001$ & 0,8460 & 0,9905 & 11,97 \\
\hline PCF (kg) & 16,08 & 14,93 & 13,34 & 12,40 & 4 & $<0,0001$ & 0,8700 & 0,9913 & 12,14 \\
\hline PPR $(\%)$ & 4,05 & 4,07 & 4,28 & 4,52 & $Y=4,23$ & 0,5073 & 0,8424 & - & 35,77 \\
\hline RCQ (\%) & 52,19 & 49,81 & 48,82 & 48,34 & 5 & 0,0123 & 0,3763 & 0,8931 & 5,97 \\
\hline $\mathrm{RCF}(\%)$ & 50,06 & 47,77 & 46,72 & 46,17 & 6 & 0,0073 & 0,3889 & 0,9122 & 5,86 \\
\hline RV (\%) & 60,84 & 59,02 & 58,93 & 58,88 & $Y=59,42$ & 0,2505 & 0,4133 & - & 5,32 \\
\hline
\end{tabular}

O PCA $(\mathrm{kg})$ apresentou comportamento linear negativo com a inclusão do feno de erva-sal na dieta (Figura 1). Pode-se inferir que a redução nos nutrientes digestíveis totais das dietas (Tabela 2) resultou em menores taxas de ganho de peso diário e, consequentemente, menores pesos ao abate dos animais. Além disso, os altos teores de fibra (59,84\% de FDNcp; Tabela 1), lignina
$(14,13 \%$; Tabela 1) e sódio $(6,13 \%$; Tabela 1) presentes na erva-sal podem ter limitado o consumo voluntário de MS, acarretando em redução do ganho de peso diário. Segundo Norman et al. (2002), a concentração de $\mathrm{NaCl}$ na ervasal é aproximadamente $17,7 \%$ da MS, sendo de 3 a $15 \%$ de sódio, o que pode constituir um fator limitante ao consumo e à digestão em ruminantes. 
Rev. Bras. Saúde Prod. Anim., Salvador, v.15, n.1, p.192-205 jan./mar., 2014 http://www.rbspa.ufba.br ISSN 15199940

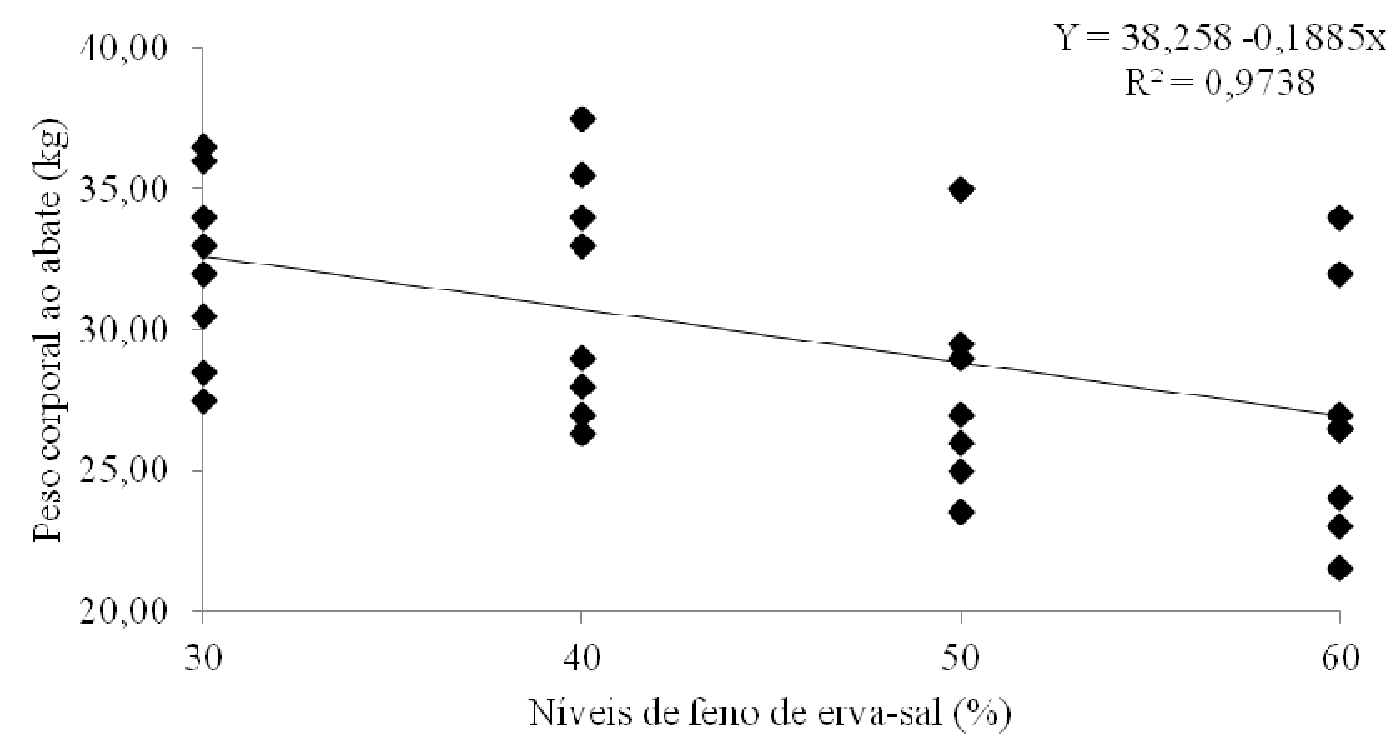

Figura 1. Efeito da inclusão de feno de erva-sal na dieta sobre o peso corporal ao abate de cordeiros Santa Inês

Os pesos e rendimentos de carcaça acompanharam a tendência do PCA e decresceram linearmente com a inclusão do feno de erva-sal. Os menores níveis de NDT (\%; Tabela 2) limitaram a síntese de músculo dos ovinos, mesmo com as exigências de proteína atendidas pela dieta. Apesar do comportamento linear negativo, os valores são considerados bastante satisfatórios, pois variaram de 48,3 a $52,2 \%$ para rendimento de carcaça quente, e de 46,2 a $50,0 \%$ para rendimento de carcaça fria, estando acima da média nacional para a raça Santa Inês, de 45\% (COSTA et al., 2010; CARTAXO et al., 2011). Além disso, o rendimento verdadeiro, que considera o peso de carcaça quente e o peso do corpo vazio e, é o mais importante para a comercialização, não foi influenciado pelos níveis de feno de erva-sal na dieta, com média de 59,4\%. O peso da carcaça é o primeiro critério a ser considerado no momento da comercialização e, juntamente com fatores como a conformação, cobertura de gordura subcutânea e cor da carne, expressam o conjunto de características utilizadas para definir uma carcaça de qualidade.

Pearce et al. (2008a), ao avaliarem as características de carcaça de borregos Merino alimentados com erva-sal e cevada ou com dieta controle, não encontraram diferenças para o rendimento de carcaça fria, com valor médio de $41,3 \%$. Estes autores relataram peso médio de carcaça quente de $17,5 \mathrm{~kg}$, similar apenas ao peso da carcaça dos animais alimentados com mais concentrado na dieta $(70 \%)$, de 16,75 $\mathrm{kg}$. Considerando que para este peso de carcaça $(16,75 \mathrm{~kg})$, o rendimento de carcaça fria foi de $50,06 \%$ enquanto o valor observado por Pearce et al. (2008a) foi de 41,3\% para peso semelhante, pode-se inferir que a superioridade no rendimento de carcaça deste trabalho deve-se ao incremento nutricional representado pelo maior nível de concentrado na dieta $(70 \%)$, o que acarreta em maior disponibilidade de energia para ganho de peso e, consequentemente, maior peso $\mathrm{e}$ rendimento de carcaça.

Não houve diferença para perdas de peso no resfriamento (PPR) e rendimento 
Rev. Bras. Saúde Prod. Anim., Salvador, v.15, n.1, p.192-205 jan./mar., 2014 http://www.rbspa.ufba.br ISSN 15199940

verdadeiro, com valores médios de 4,23 e $59,42 \%$, respectivamente. A PPR está ligada principalmente à uniformidade da cobertura de gordura subcutânea, que protege a carcaça durante o resfriamento contra perdas de água e, consequentemente, de peso. Baixa PPR é desejada pelos frigoríficos e também pelos varejistas que comercializam carne ovina, pois este parâmetro confere maior rendimento final na carcaça e nos cortes. Em ovinos, a PPR varia de 1 a $7 \%$ e é influenciada pela uniformidade da gordura de cobertura, sexo do animal, temperatura e umidade relativa da câmara frigorífica (MUELA et al., 2010), sendo que os valores encontrados neste trabalho $(4,23 \%)$ estão condizentes com os da literatura. Apesar de não ter havido diferença estatística, houve tendência $(\mathrm{P}=0,5073)$ de aumento das PPR quando o nível de feno de erva-sal aumentou na dieta, podendo estar relacionado à redução da espessura de gordura subcutânea nesses animais, observada pela Tabela 5, e que estaria envolvido no aumento das perdas por resfriamento, já que a gordura na carcaça atua como protetor contra perdas de água.

Pela Tabela 4, observa-se que houve efeito linear decrescente para os pesos $(\mathrm{kg})$ de todos os cortes da carcaça, sendo maiores nos animais alimentados com $30 \%$ de feno de erva-sal e $70 \%$ de concentrado.

Esses resultados estão de acordo com a premissa de que o peso dos cortes comerciais aumenta à medida que o peso absoluto de carcaça também aumenta (SANTOS-CRUZ et al., 2009).

Tabela 4. Pesos e rendimentos dos cortes comerciais das carcaças de cordeiros alimentados com níveis crescentes de feno de erva-sal e concentrado

\begin{tabular}{|c|c|c|c|c|c|c|c|c|c|}
\hline \multirow{2}{*}{ Variável } & \multicolumn{4}{|c|}{ Níveis de feno de erva-sal (\%) } & \multirow{2}{*}{ Equação de regressão } & \multicolumn{2}{|c|}{ Valor de P } & \multirow{2}{*}{$\mathrm{R}^{2}$} & \multirow{2}{*}{$\begin{array}{l}\text { CV } \\
(\%)\end{array}$} \\
\hline & 30 & 40 & 50 & 60 & & $\mathrm{~L}$ & Q & & \\
\hline \multicolumn{10}{|l|}{ Paleta } \\
\hline $\mathrm{Kg}$ & 1,53 & 1,38 & 1,30 & 1,23 & 1 & 0,0036 & 0,5858 & 0,9673 & 14,42 \\
\hline$\%$ & 19,24 & 18,80 & 19,41 & 19,61 & $Y=19,26$ & 0,4968 & 0,5763 & - & 8,18 \\
\hline \multicolumn{10}{|l|}{ Pescoço } \\
\hline $\mathrm{Kg}$ & 0,65 & 0,63 & 0,63 & 0,52 & 2 & 0,0413 & 0,4174 & 0,8248 & 19,46 \\
\hline$\%$ & 8,20 & 8,39 & 9,14 & 8,36 & $Y=8,52$ & 0,5283 & 0,2612 & - & 14,09 \\
\hline \multicolumn{10}{|l|}{ Costelas } \\
\hline $\mathrm{Kg}$ & 2,08 & 2,05 & 1,79 & 1,61 & 3 & 0,0003 & 0,4415 & 0,9362 & 13,43 \\
\hline$\%$ & 26,30 & 27,65 & 26,57 & 25,77 & $Y=26,57$ & 0,2787 & 0,0556 & - & 5,75 \\
\hline \multicolumn{10}{|l|}{ Lombo } \\
\hline $\mathrm{Kg}$ & 0,96 & 0,89 & 0,75 & 0,68 & 4 & $<0,001$ & 0,9841 & 0,9843 & 15,80 \\
\hline$\%$ & 12,15 & 11,92 & 11,20 & 10,83 & 5 & 0,0298 & 0,8834 & 0,9661 & 11,22 \\
\hline \multicolumn{10}{|l|}{ Perna } \\
\hline $\mathrm{Kg}$ & 2,70 & 2,43 & 2,25 & 2,20 & 6 & 0,0022 & 0,3307 & 0,9185 & 13,11 \\
\hline$\%$ & 33,96 & 32,94 & 33,60 & 35,03 & $Y=33,88$ & 0,2138 & 0,0727 & - & 5,70 \\
\hline
\end{tabular}

No entanto, Mattos (2009) não encontrou diferenças nos rendimentos dos principais cortes da carcaça de cordeiros, perna $(31,9 \%)$, paleta $(19,8 \%)$ e lombo $(9,9 \%)$, ao avaliar o efeito de diferentes níveis de feno de 
erva-sal e palma forrageira na terminação de cordeiros Santa Inês.

Entretanto, para os rendimentos (\%) de cortes, houve efeito linear apenas para o rendimento de lombo, que decresceu à medida que houve maior proporção de feno na dieta. O lombo tem crescimento tardio em relação à carcaça do animal, pois as ondas de crescimento partem das extremidades do corpo em direção ao seu centro. Pode-se inferir que o decréscimo no teor de energia das dietas afetou mais marcadamente o lombo, em relação à carcaça, por se tratar de uma zona de crescimento tardio e, portanto, mais suscetível à partição limitante de energia (GERRARD \& GRANT, 2006; SANTOS-CRUZ et al., 2009).

Apesar das diferenças nos pesos e rendimentos de carcaça, a porcentagem dos cortes comerciais, exceto a de lombo, não foi influenciada pelas diferentes dietas, o que constitui um resultado interessante, já que a tendência atual dos sistemas de venda de carne ovina é utilizar os cortes de carcaça e cortes cárneos como unidades de comercialização em detrimento das carcaças. Estes cortes em peças individualizadas, associados à apresentação do produto, são importantes fatores na comercialização, pois além dos preços diferenciados, permitem melhor aproveitamento da carne.

$\mathrm{Na}$ Tabela 5, nota-se que a profundidade do músculo Longissimus lumborum (medida B), espessuras mínima (EG) e máxima (GR) de gordura e área de olho de lombo (AOL) foram reduzidas à medida que houve aumento do nível de feno de erva-sal na dieta. Os valores de espessura de gordura obtidos no presente estudo são considerados baixos em relação aos encontrados para animais de raças mais especializadas na produção de carne, como Dorper, Texel e Ile de France. A espessura mínima de gordura almejada pelos frigoríficos de cordeiros varia de 3 a $5 \mathrm{~mm}$, sendo que acima deste valor, pode haver aumento dos custos de produção para os produtores, e também para frigoríficos e processadores de carne que precisam realizar uma toalete nos cortes, retirando o excesso de gordura. Neste estudo, nenhum dos níveis de erva-sal avaliados proporcionou a espessura mínima de gordura preconizada para cordeiros, de $3 \mathrm{~mm}$ (Figura 2).

Tabela 5. Medidas do músculo Longissimus lumborum, espessura mínima (EG) e máxima de gordura (GR) e área de olho de lombo (AOL) em cordeiros alimentados com níveis crescentes de feno de erva-sal e concentrado

\begin{tabular}{|c|c|c|c|c|c|c|c|c|c|}
\hline \multirow{2}{*}{ Variável } & \multicolumn{4}{|c|}{ Níveis de feno de erva-sal (\%) } & \multirow{2}{*}{$\begin{array}{l}\text { Equação de } \\
\text { regressão }\end{array}$} & \multicolumn{2}{|c|}{ Valor de P } & \multirow[b]{2}{*}{$\mathrm{R}^{2}$} & \multirow{2}{*}{$\begin{array}{l}\text { CV } \\
(\%)\end{array}$} \\
\hline & 30 & 40 & 50 & 60 & & $\mathrm{~L}$ & Q & & \\
\hline Medida $A^{a}$ & 5,28 & 5,06 & 5,20 & 4,94 & $\mathrm{Y}=5,12$ & 0,1609 & 0,8780 & - & 7,38 \\
\hline Medida $B^{b}$ & 2,88 & 2,83 & 2,58 & 2,51 & 1 & 0,0005 & 0,8651 & 0,9274 & 8,11 \\
\hline $\mathrm{EG}(\mathrm{mm})$ & 1,78 & 1,74 & 1,25 & 1,05 & 2 & 0,0044 & 0,6729 & 0,9120 & 37,61 \\
\hline GR (mm) & 3,90 & 4,03 & 2,81 & 2,13 & 3 & 0,0028 & 0,3815 & 0,8651 & 39,42 \\
\hline $\operatorname{AOL}\left(\mathrm{cm}^{2}\right)^{\mathrm{c}}$ & 11,90 & 11,26 & 10,55 & 9,80 & 4 & 0,0039 & 0,9812 & 0,9999 & 13,50 \\
\hline
\end{tabular}

${ }^{a}$ Medida $\mathrm{A}=$ comprimento máximo do músculo Longissimus lumborum, $\mathrm{em} \mathrm{cm} ;{ }^{\mathrm{b}}$ Medida $\mathrm{B}=$ profundidade máxima do músculo Longissimus lumborum, em $\mathrm{cm} ;{ }^{\mathrm{C}} \mathrm{AOL}=(\mathrm{A} / 2 \times \mathrm{B} / 2) \pi$, Costa et al (2012).

${ }^{1} \mathrm{Y}=3,31-0,016 \mathrm{x} ;{ }^{2} \mathrm{Y}=0,26-0,003 \mathrm{x} ;{ }^{3} \mathrm{Y}=0,62-0,006 \mathrm{x} ;{ }^{4} \mathrm{Y}=14,18-0,07 \mathrm{x}$. 


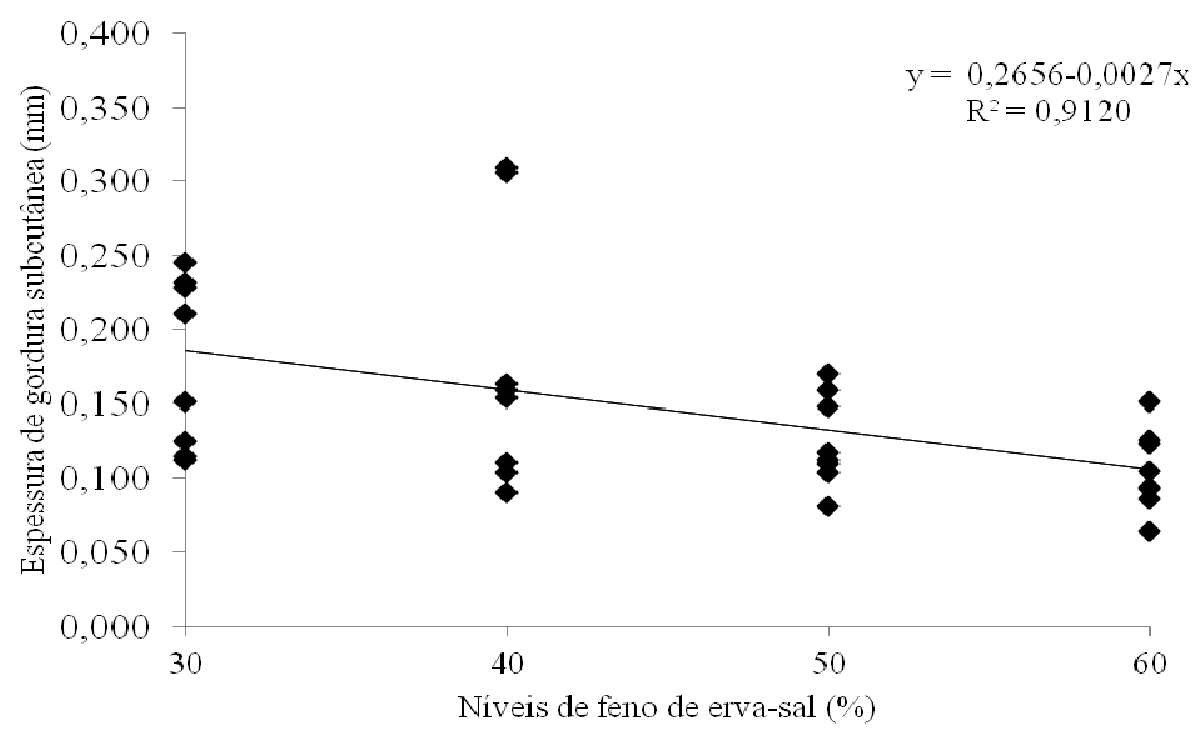

Figura 2. Efeito da inclusão de feno de erva-sal na dieta sobre a espessura mínima de gordura subcutânea na carcaça de cordeiros Santa Inês

Alguns trabalhos têm demonstrado que o fornecimento de erva-sal para ovinos em terminação pode estar correlacionado à redução da quantidade de gordura nas carcaças produzidas. Pearce et al. (2008a) verificaram menor proporção de gordura e maior de músculo na carcaça de borregos Merino alimentados em confinamento com erva-sal suplementada com cevada em relação aos que receberam dieta controle (cevada + feno de aveia). Pearce et al. (2008b) também observaram redução da espessura de gordura subcutânea em cordeiros pastejando erva-sal $(1,4 \mathrm{~mm})$ quando comparados aos que pastejavam forragem senescente e palha de cevada $(2,1 \mathrm{~mm})$. Segundo os autores supracitados, o alto consumo de sal pode afetar a relação proteína:energia da dieta e acarretar em menor disponibilidade de energia para deposição no corpo do animal na forma de gordura. Além disso, o aumento do gasto energético para metabolizar e excretar grandes quantidades de sódio no organismo pode reduzir a disponibilidade de energia para lipogênese (ARIELI et al., 1989). Se a exigência de energia não for atendida pela alimentação, o tecido adiposo pode ser usado para fornecer os substratos energéticos, resultando em declínio nas reservas de gordura do animal.

Outro fator interessante é a grande quantidade do aminoácido betaína na erva-sal $(30 \mathrm{~g} / \mathrm{kg}$ MS; BEN SALEM et al., 2010; JOSEPH et al., 2013), que pode estar envolvido nos mecanismos de redução da gordura corporal em animais alimentados com esta planta, pois Ratrivanto et al. (2009), revisaram os efeitos da betaína no metabolismo animal e constataram redução da gordura corporal. Pearce et al. (2008a) avaliaram o efeito do pastejo de ovinos em erva-sal sobre alguns hormônios metabólicos e observaram menor concentração de insulina e maior de hormônio do crescimento $(\mathrm{GH})$ nestes animais, em relação aos que receberam dieta controle sem erva-sal. Estes autores explicam que a insulina é o principal hormônio regulador dos depósitos de gordura corporal e, quando 
animais são alimentados com mais energia digestível na dieta há aumento da produção de propionato que, por sua vez, estimula a síntese de insulina e induz a deposição de gordura. Embora não se tenha determinado as concentrações de insulina neste estudo, este pode ser um dos fatores envolvidos na redução da EG subcutânea nos cordeiros alimentados com maiores proporções de feno de erva-sal (HOCQUETTE et al., 2010).

A área de olho de lombo (AOL) foi linearmente reduzida à medida que o nível de feno de erva-sal na dieta aumentou. Esta medida é utilizada na predição da quantidade de músculo da carcaça, pois o Longissimus lumborum é de maturidade tardia e de fácil mensuração, sendo usado como indicador da musculosidade das carcaças. Como houve redução no peso das carcaças dos animais que receberam maiores quantidades de erva-sal (Tabela 3), a AOL seguiu esta tendência, já que está relacionada ao peso do animal e, consequentemente, da carcaça como um todo.

Nas Tabelas 6 e 7, observam-se as médias dos pesos e porcentagens dos não-componentes da carcaça, em relação ao PCA, respectivamente. Houve efeito linear decrescente para pesos de pele e coração, pesos e rendimentos de fígado, rins com gordura perirrenal, gorduras omental e mesentérica, à medida que o nível de feno na dieta aumentou. Órgãos como pele, fígado e coração têm crescimento isogônico em relação ao peso corporal do animal (SANTOS-CRUZ et al., 2009). Assim, o decréscimo nos níveis de energia reduziu o peso corporal ao abate e, dessa forma, o peso de alguns constituintes do peso corporal dos ovinos.

Tabela 6. Pesos dos não-componentes da carcaça de cordeiros alimentados com níveis crescentes de feno de erva-sal e concentrado

\begin{tabular}{|c|c|c|c|c|c|c|c|c|c|}
\hline \multirow{2}{*}{ Variável (kg) } & \multicolumn{4}{|c|}{ Níveis de feno de erva-sal (\%) } & \multirow{2}{*}{$\begin{array}{l}\text { Equação de } \\
\text { regressão }\end{array}$} & \multicolumn{2}{|c|}{ Valor de $\mathrm{P}$} & \multirow{2}{*}{$\mathrm{R}^{2}$} & \multirow{2}{*}{$\begin{array}{l}\text { CV } \\
(\%)\end{array}$} \\
\hline & 30 & 40 & 50 & 60 & & $\mathrm{~L}$ & Q & & \\
\hline TGI $^{\mathrm{a}}$ & 2,17 & 2,27 & 2,09 & 1,91 & $\mathrm{Y}=2,11$ & 0,1140 & 0,2143 & - & 18,14 \\
\hline $\begin{array}{l}\text { Conteúdo do } \\
\text { TGI }\end{array}$ & 4,61 & 4,92 & 4,93 & 4,81 & $\mathrm{Y}=4,82$ & 0,7060 & 0,4628 & - & 20,54 \\
\hline Sangue & 1,24 & 1,17 & 1,09 & 1,03 & $Y=1,13$ & 0,0508 & 0,8804 & - & 19,62 \\
\hline Pele & 2,71 & 2,59 & 2,31 & 2,12 & 1 & 0,0050 & 0,5584 & 0,939 & 18,94 \\
\hline Esôfago & 0,05 & 0,05 & 0,05 & 0,05 & $Y=0,05$ & 0,4441 & 0,8070 & - & 24,36 \\
\hline Fígado & 0,66 & 0,65 & 0,54 & 0,47 & 2 & 0,0015 & 0,3655 & 0,994 & 20,88 \\
\hline Coração & 0,23 & 0,21 & 0,17 & 0,17 & 3 & 0,0006 & 0,9646 & 0,678 & 22,86 \\
\hline Ap. respiratório & 0,62 & 0,61 & 0,59 & 0,54 & $Y=0,59$ & 0,3184 & 0,8102 & - & 21,65 \\
\hline Cabeça & 1,34 & 1,34 & 1,28 & 1,28 & $Y=1,31$ & 0,2293 & 0,7444 & - & 10,37 \\
\hline Patas & 0,72 & 0,70 & 0,67 & 0,66 & $Y=0,69$ & 0,0731 & 0,7662 & - & 13,75 \\
\hline Baço & 0,07 & 0,07 & 0,06 & 0,06 & $Y=0,06$ & 0,2347 & 0,9116 & - & 34,49 \\
\hline $\begin{array}{l}\text { Rins + gord. } \\
\text { per, }\end{array}$ & 0,42 & 0,40 & 0,31 & 0,23 & 4 & 0,0037 & 0,4132 & 0,896 & 34,83 \\
\hline Gord. omental $^{\mathrm{c}}$ & 0,76 & 0,61 & 0,35 & 0,30 & 5 & $<0,001$ & 0,6614 & 0,989 & 43,01 \\
\hline Gord. Mesent. $^{\mathrm{d}}$ & 0,47 & 0,38 & 0,32 & 0,30 & 6 & 0,0006 & 0,6066 & 0,719 & 27,71 \\
\hline
\end{tabular}

${ }^{\mathrm{a}}$ Trato gastrintestinal; ${ }^{\mathrm{b}}$ rins com gordura perirrenal; ${ }^{\mathrm{c}}$ gordura omental (recobre os estômagos); ${ }^{\mathrm{d}}$ gordura mesentérica (recobre os intestinos).

${ }^{1} \mathrm{Y}=3,47-0,02 \mathrm{x} ;{ }^{2} \mathrm{Y}=0,90-0,007 \mathrm{x} ;{ }^{3} \mathrm{Y}=0,32-0,002 \mathrm{x} ;{ }^{4} \mathrm{Y}=0,63-0,006 \mathrm{x} ;{ }^{5} \mathrm{Y}=1,26-0,02 \mathrm{x} ;{ }^{6} \mathrm{Y}=0,65-$ $0,005 x$. 
Rev. Bras. Saúde Prod. Anim., Salvador, v.15, n.1, p.192-205 jan./mar., 2014 http://www.rbspa.ufba.br ISSN 15199940

Tabela 7. Rendimentos (\%) dos não-componentes da carcaça em relação ao peso corporal ao abate de cordeiros alimentados com níveis crescentes de feno de erva-sal e concentrado

\begin{tabular}{|c|c|c|c|c|c|c|c|c|c|}
\hline \multirow{2}{*}{ Variável (\%) } & \multicolumn{4}{|c|}{ Níveis de feno de erva-sal (\%) } & \multirow{2}{*}{$\begin{array}{l}\text { Equação de } \\
\text { regressão }\end{array}$} & \multicolumn{2}{|c|}{ Valor de P } & \multirow{2}{*}{$\mathrm{R}^{2}$} & \multirow{2}{*}{$\begin{array}{l}\text { CV } \\
(\%)\end{array}$} \\
\hline & 30 & 40 & 50 & 60 & & $\mathrm{~L}$ & $\mathrm{Q}$ & & \\
\hline TGI & 6,73 & 7,18 & 7,23 & 7,18 & $\mathrm{Y}=7,08$ & 0,2713 & 0,264 & - & 9,98 \\
\hline $\begin{array}{l}\text { Conteúdo do } \\
\text { TGI }^{\mathrm{a}}\end{array}$ & 14,25 & 15,60 & 17,06 & 17,97 & 1 & 0,0005 & 0,755 &, 8981 & 10,44 \\
\hline Sangue & 3,39 & 3,32 & 3,30 & 3,32 & $Y=3,33$ & 0,7111 & 0,767 & - & 10,03 \\
\hline Pele & 7,45 & 7,42 & 7,02 & 6,82 & 2 & 0,0294 & 0,4700 & 0,6912 & 10,64 \\
\hline Esôfago & 0,14 & 0,14 & 0,14 & 0,16 & $\mathrm{Y}=0,14$ & 0,5852 & 0,6158 & - & 25,76 \\
\hline Fígado & 1,80 & 1,84 & 1,63 & 1,51 & 3 & 0,0006 & 0,1408 & 0,8447 & 10,26 \\
\hline Coração & 0,64 & 0,59 & 0,52 & 0,54 & 4 & 0,0090 & 0,9439 & 0,9834 & 19,67 \\
\hline Ap. Respiratorio & 1,69 & 1,79 & 1,79 & 1,75 & $\mathrm{Y}=1,75$ & 0,4193 & 0,975 & - & 16,63 \\
\hline Cabeça & 3,70 & 3,90 & 3,92 & 4,15 & 5 & 0,0058 & 0,838 & 0,9440 & 7,35 \\
\hline Patas & 1,99 & 2,00 & 2,07 & 2,12 & $\mathrm{Y}=2,05$ & 0,3130 & 0,930 & - & 9,60 \\
\hline Baço & 0,19 & 0,19 & 0,18 & 0,18 & $\mathrm{Y}=0,18$ & 0,7851 & 0,982 & - & 25,12 \\
\hline $\begin{array}{l}\text { Rins + gord. } \\
\text { per, }{ }^{\text {b }}\end{array}$ & 1,15 & 1,15 & 0 , & 0 , & 6 & 20 & 97 & 53 & 31,96 \\
\hline Gord. omental $^{\mathrm{c}}$ & 1,23 & 1,10 & 0,97 & 0,96 & 7 & 0,0004 & 0,660 & 0,8675 & 28,17 \\
\hline Gord. Mesent. $^{\mathrm{d}}$ & 2,07 & 1,70 & 1,08 & 1,01 & 8 & 0,0006 & 0,525 & 0,9992 & 40,24 \\
\hline
\end{tabular}

a conteúdo do trato gastrintestinal; ${ }^{b}$ rins com gordura perirrenal; ${ }^{c}$ gordura omental (recobre os estômagos); d gordura mesentérica (recobre os intestinos).

${ }^{1} \mathrm{Y}=10,49+0,01 \mathrm{x} ;{ }^{2} \mathrm{Y}=8,43-0,02 \mathrm{x} ;{ }^{3} \mathrm{Y}=2,19-0,01 \mathrm{x} ;{ }^{4} \mathrm{Y}=0,80-0,004 \mathrm{x} ;{ }^{5} \mathrm{Y}=3,28+0,01 \mathrm{x} ;{ }^{6} \mathrm{Y}=1,60-$ $0,01 \mathrm{x} ;{ }^{7} \mathrm{Y}=1,65-0,009 \mathrm{x} ;{ }^{8}$.

A inclusão do feno de erva-sal resultou provavelmente em menor produção de propionato no rúmen dos animais. $\mathrm{O}$ propionato é o principal precursor neoglicogênico do metabolismo dos ruminantes, porém, essa rota metabólica também pode ser suprida por glicerol e aminoácidos, por exemplo. Provavelmente, a elevação do nível de erva-sal reduziu o propionato circulante aumentando a demanda por outras fontes neoglicogênicas como o glicerol, culminando em lipólise e baixa deposição de massa adiposa no corpo do animal (HOMEM JÚNIOR et al., 2010; BERNES et al., 2012; BERNES \& STENGÄRDE, 2012). Essas deposições também estão associadas a efeitos genéticos e ambientais que ocorrem em ovinos e caprinos tropicais, principalmente, como reserva energética quando há disponibilidade quantitativa e qualitativa de alimentos, para serem mobilizadas durante o período de escassez (MIRKENA et al., 2010). É importante ressaltar que o acúmulo de grandes quantidades de gordura visceral não é desejado, pois há aumento das exigências de energia para mantença, devido à maior taxa metabólica do tecido adiposo e há desperdício da energia fornecida pela dieta, já que esta gordura não é aproveitada para consumo humano (TOLDRÁ et al. 2012).

A maior inclusão de volumoso na dieta aumentou a proporção de conteúdo do trato gastrintestinal (TGI) dos animais, já que este tipo de alimento necessita de maior tempo de retenção no rúmen para sua degradação (Tabela 7).

Segundo Van Soest (1994), o fígado é responsável pela captação de cerca de $80 \%$ do propionato que passa pelo 
sistema portal, para a conversão em glicose, e pela captação da amônia e sua conversão em ureia, além da síntese e degradação de aminoácidos (ALLEN \& BRADFORD, 2012). Neste sentido, a redução dos níveis de concentrado e a consequente redução dos teores de energia das dietas (Tabela 1), embora discretos, podem ter causado menor desenvolvimento de fígado e coração, o que não ocorreu com todos os outros órgãos.

O aumento da proporção de feno de ervasal na dieta de cordeiros proporciona adequados rendimentos verdadeiros de carcaça e de cortes comerciais, no entanto, reduz a área de olho de lombo e a espessura de gordura subcutânea, medidas importantes na avaliação qualitativa da carcaça ovina.

\section{AGRADECIMENTOS}

À Fundação de Amparo à Pesquisa do Estado de São Paulo (FAPESP) e ao Banco Nacional do Desenvolvimento (BNDES), pelo suporte financeiro.

\section{REFERÊNCIAS}

ALLEN, M.S.; BRADFORD, B.J.

Control of food intake by metabolism of fuels: a comparison across species. The Proceedings of the Nutrition Society, v.71, n.3, p.401-409, 2012.

ARIELI, A.; NAIM, E.; BENJAMIN, R.; PASTERNAK, D. The effect of feeding saltbush and sodium chloride on energy metabolism in sheep. Animal Production, v.49, n.3, p.451-457, 1989.
BEN SALEM, H.; NORMAN, H.C.; NEFZAOUI, A.; MAYBERRY, D.E.; PEARCE, K.L.; REVELL, D.K. Potential use of oldman saltbush (Atriplex nummularia Lindl.) in sheep and goat feeding. Small Ruminant Research, v.91, n.1, p.13-28, 2010.

BERNES, G.; STENGÄRDE, L. Sheep fed only silage or silage supplemented with concentrates. 1. Effects on ewe performance and blood metabolites. Small Ruminant Research, v.102, n.2, p.108-113, 2012.

BERNES, G.; TURNER, T.; PICKOVA, J. Sheep fed only silage or silage supplemented with concentrates 2 . Effects on lamb performance and fatty acid profile of ewe milk and lamb meat. Small Ruminant Research, v.102, n.2, p.114 124, 2012.

CARTAXO, F.Q.; SOUSA, W.H.; COSTA, R.G.; CEZAR, M.F.; PEREIRA FILHO, J.M.; CUNHA, M.G.G.

Quantitative traits of carcass from lambs of different genotypes submitted to two diets. Revista Brasileira de Zootecnia, v.40, n.10, p.2220-2227, 2011.

COLOMER-ROCHER, F.; DELFA, R.; SIERRA, I. Método normalizado para el estudio de los caracteres cuantitativos y cualitativos de las canales, según los sistemas de produccíon. In: COLOMERROCHER, F.; DELFA, R.; SIERRA, I. Método normalizado para el estudio de los caracteres cuantitativos y cualitativos de las canales caprinas y ovinas. Madrid: INIA, 1988. p.19-41.

COSTA, R.G.; ARAÚJO FILHO, J.T.; SOUSA, W.H.; GONZAGA NETO, S.; MADRUGA, M.S.; FRAGA, A.B. Effect of diet and genotype on carcass characteristics of feedlot hair sheep.

Revista Brasileira de Zootecnia, v.39, n.12, p.2763-2768, 2010. 
Rev. Bras. Saúde Prod. Anim., Salvador, v.15, n.1, p.192-205 jan./mar., 2014 http://www.rbspa.ufba.br ISSN 15199940

COSTA, R.G.; LIMA, A.G.V.O.; OLIVEIRA, C.F.S.; AZEVEDO, P.S.; MEDEIROS, A.N. Using different methodologies to determination the loin eye area in sheep. Archivos de

Zootecnia, v.61, n.6, p.615-618, 2012.

El SHAER, H.M. Halophytes and salttolerant plants as potential forage for ruminants in the Near East region.

Small Ruminant Research, v.91, n.10, p.3-12, 2010.

FERNANDEZ, C.; LOPEZ-SAEZ, A.; GALLEGO, L.; DE LA FUENTE, J. M. Effect of source of betaine on growth performance and carcass traits in lambs. Animal Feed Science and Technology, v.86, n.1-2, p.71-82, 2000.

GERRARD, D.E.; GRANT, A.L.

Principles of animal growth and development. Kendall/Hunt Publishing Company, 2006. 264p.

GARDNER, G.E.; PETHICK, D.W.; SMITH, G. Effect of chromium chelavite supplementation on the metabolism of glycogen and lipid in adult merino sheep. Australian Journal of Agricultural Research, v.49, n.1, p.137-145, 1998.

HOCQUETTE, J.F.; GONDRET, F.; BAÈZA, E.; MÈDALE, F.; JURIE, C.; PETHICK, D.W. Intramuscular fat content in meat-producing animals: development, genetic and nutritional control, and identification of putative markers. Animal, v.4, n.2, p.303-319, 2010.

HOMEM JUNIOR, A.C.; EZEQUIEL, J.M.B.; FÁVARO, V.R.; OLIVEIRA, P.S.N.; D'AUREA, A.P.; SANTOS, V.C.; GONÇALVES, J.S. Ruminal fermentation of sheep fed highconcentrated diet containing sunflower grains or protected fat. Arquivo
Brasileiro de Medicina Veterinária e

Zootecnia, v.62, n.1, p.144-153, 2010.

JOSEPH, S.; MURPHY, D.; BHAVE, $\mathrm{M}$. Glycine betaine biosynthesis in saltbushes (Atriplex spp.) under salinity stress. Biologia, v.68, n.5, p.879-895, 2013.

MATTOS, C.W. Associação de palma forrageira (Opuntia ficus-indica Mill) e feno de erva-sal (Atriplex nummularia L.) em dietas para cordeiros Santa Inês em confinamento. 2009.101 f. Tese (Doutorado em Zootecnia) Universidade Federal Rural de Pernambuco, Recife.

MENESES, R.; VARELA, G.; FLORES, H. Evaluating the use of Atriplex nummularia hay on feed intake, growth, and carcass characteristics of creole kids. Chilean Journal of Agricultural Research, v.72, n.1, p.7479, 2012.

MIRKENA, T.; DUGUMA, G.; HAILE, A.; TIBBO, M.; OKEYO, A. M.; WURZINGER, M.; SÖLKNER, J. Genetics of adaptation in domestic farm animals: a review. Livestock Science, v.132, n.1-3., p.1-12, 2010.

MUELA, E.; SAÑUDO, C.; CAMPO, M.M.; MEDEL, I.; BELTRÁN, J.A. Effects of cooling temperature and hot carcass weight on the quality of lamb. Meat Science, v.84, n.1, p.101-107, 2010.

NATIONAL RESEARCH COUNCIL NRC. Nutrient requirements of small ruminants. Washington: The National Academy Press, 2007. 325p.

NORMAN, H.C.; DYNES, R.A.; MASTERS, D.G. Nutritive value of plants growing on saline land. In: 
Rev. Bras. Saúde Prod. Anim., Salvador, v.15, n.1, p.192-205 jan./mar., 2014 http://www.rbspa.ufba.br ISSN 15199940

NATIONAL CONFERENCE AND WORKSHOP ON THE PRODUCTIVE USE AND REHABILITATION OF

SALINE LANDS, 8th., 2002, Fremantle. Proceedings... Fremantle: Canning Bridge, 2002. p.59-69.

PEARCE, K.L.; PETHICK, D.W.; MASTERS, D.G. The effect of ingesting a saltbush and barley ration on the carcass and eating quality of sheepmeat. Animal, v.2, n.3, p.479490, 2008a.

PEARCE, K.L.; NORMAN, H.C.; WILMOT, M.; RINTOUL, A.; PETHICK, D.W.; MASTERS, D.G. The effect of grazing saltbush with a barley supplement on the carcass and eating quality of sheep meat. Meat Science, v.79, n.2, p.344-454, 2008 b.

PEARCE, K.L.; NORMAN, H.C.; HOPKINS, D.L. The role of saltbushbased pasture systems for the production of high quality sheep and goat meat. Small Ruminant Research, v.91, n.1, p.29-38, 2010.

RATRIYANTO, A.; MOSENTHIN, R.; BAUER, E.; EKLUND, M. Metabolic, osmoregulatory and nutritional functions of betaine in monogastric animals. Asian-Australian Journal of Animal Science, v.22, n.10, p.14611476, 2009.

SANTOS-CRUZ, C.L.; PÉREZ, J.R.O.; MUNIZ, J.A.; CRUZ, C.A.C.; ALMEIDA, T.R.V. Development of live weight components from Santa Inês and Bergamácia lambs slaughtered at different weights. Revista Brasileira de Zootecnia, v.38, n.5, p.923-932, 2009.
STATISTICAL ANALYSIS SYSTEM. SAS system for windows. Cary: SAS Institute, 2002.

TOLDRÁ, F.; CONCEPCIÓN ARISTOY, M.; MORA, L.; REIG, M. Innovations in value-addition of edible meat by-products. Meat Science, v.92, n.2, p.290-296, 2012.

Van NIEKERK, W.A.; HASSEN, A.; VERMAAK, P.J.; COERTZE, R.J. Influence of species/cultivar and season on the quality of Atriplex grown at different sites in South Africa. South African Journal of Animal Science, v.39, p.238-241, 2009.

Van SOEST, P.J. Nutritional ecology of the ruminant. 2 nd. ed. Ithaca: Cornell University Press, 1994. 476p.

WEISS, W.P. Energy prediction equations for ruminant feeds. In: CORNELL NUTRITION CONFERENCE FEED MANUFACTUREs, 61, 1999, Ithaca. Proceedings... Ithaca: Cornell University, 1999. p.176-185.

Data de recebimento: 15/10/2013

Data de aprovação: 26/03/2014 